\title{
Multidimensional single-cell benchmarking of inducible promoters for precise dynamic control in budding yeast
}

\author{
Vojislav Gligorovski ${ }^{1}$, Ahmad Sadeghi ${ }^{1}$ and Sahand Jamal Rahi ${ }^{1}$ \\ ${ }^{1}$ Laboratory of the Physics of Biological Systems, Institute of Physics, École polytechnique fédérale de \\ Lausanne (EPFL), Lausanne, Switzerland
}

\begin{abstract}
For quantitative systems biology, simultaneous readout of multiple cellular processes as well as precise, independent control over different genes' activities are needed. In contrast to readout systems such as fluorescent proteins, control systems such as inducible transcription-factor-promoter systems have not been characterized systematically, impeding reliable modeling and precise system-level probing of biological systems.

We built a comprehensive single-copy library of inducible promoters controlling fluorescent protein (yEVenus) expression in budding yeast, including GAL1pr, GALLpr, MET3pr, CUP1pr, PHO5pr, tetOpr, terminator-tetOpr and the blue light-inducible systems EL222-LIP, EL222-GLIP. To track their properties under dynamic perturbations, we performed high-throughput time-lapse microscopy. The analysis of $>100000$ cell images was made possible by the recently developed convolutional neural network YeaZ. We report key coarse-grained kinetic parameters, levels of noise, and effects on cellular growth. Our multidimensional benchmarking uncovers unexpected disadvantages of widely used tools, e.g., slow off kinetics of the doxycycline-induced tetOpr system, nomonotonic activity, or high variability of PHO5pr. Our data would guide the choice of acceptable compromises for applications. Evaluating the ARG3 promoter for potential use as a new inducible system, we discovered that it has an interesting OR gate function and that it turns on in the presence of methionine in synthetic complete medium. To demonstrate the ability to finely control genetic circuits, we tuned the time between cell cycle Start and mitotic entry in budding yeast experimentally, exogenously simulating near-wild-type timing.

The data presented here ought to facilitate the choices of expression systems for quantitative experiments and applications in systems and synthetic biology and to serve as a reference to benchmark new inducible systems.
\end{abstract}




\section{Introduction}

Control over the level and timing of gene activity does not only offer advantages over more traditional genetics approaches such as gene knockout or overexpression but is indispensable for many applications. In particular, understanding the organization and function of system-level properties as well as constructing novel artificial cellular behaviors frequently requires the temporally precise, reversible manipulation of several nodes in a gene network at once. As a result, inducible expression systems and their characterization are critical for advances in systems and synthetic biology.

Inducible system have been widely applied in systems biology for studying the dynamics, topology, and stochasticity of genetic networks. ${ }^{1-3}$ In metabolic engineering, these systems are employed for the reversible activation of biosynthetic pathways at specific stages of growth or for fine-tuning activation levels. ${ }^{4-6}$ Reversible activation of gene activity is also needed in synthetic biology for the construction of switchable logic circuits ${ }^{7,8}$ or to reduce the toxic effects of specific gene products 9 .

Exogenous regulation of gene expression in eukaryotes can be introduced at different stages: at the transcriptional or translational level as well as at the posttranslational level by controlling protein-protein interactions or protein degradation. ${ }^{10,11}$ In Saccharomyces cerevisiae, a widely used organism in research and industrial applications, the most common way of tuning the level of gene expression is by regulating transcription. ${ }^{12}$ Moreover, the majority of the tools for manipulating gene expression, including inducible promoters, have been engineered for yeasts. ${ }^{13}$

Many commonly used inducible promoters in budding yeast are regulated by small metabolites, such as galactose, methionine, or copper. ${ }^{14}$ Using nutrients in order to control genes has the drawback that changes in nutrient levels generally also affect metabolism. To avoid such effects, synthetic systems have been developed, which are based on transcription factors induced by compounds not naturally present in the host, such as tetracycline ${ }^{15}$ More recently, light sensors from bacteria and plants have been adapted for use in budding yeast. ${ }^{4,16}$ In contrast to the other systems for manipulating cellular processes, light in principle provides a rapid, noninvasive, and easily switchable means of control. ${ }^{17}$ Although the synthetic systems do not directly interact with cellular physiology, they can nevertheless have an effect on cellular growth due to the toxicity of the inducer, for example.

Precise control of gene activity requires inducible systems that ideally have fast kinetics, low basal activity (leakiness), and low noise. However, for inducible systems used in budding yeast, most of these properties have not been assessed precisely, not in a manner that would allow their direct comparison, or have not been determined at all. Due to a lack of quantitative description, the selection of the inducible system is usually guided by intuition or trial and error.

There are at least two technical difficulties for quantitatively characterizing inducible systems:

1) To assess the variability of many dynamic properties, single-cell time courses need to be recorded by fluorescence microscopy and analyzed. Taking snapshots of the population by flow cytometry does not suffice for reconstructing time courses unambiguously. For this to be feasible with sufficient numbers of cells, a highly efficient and accurate segmentation method such as the newly developed convolutional neural network YeaZ ${ }^{19}$ was needed, which we used to analyze $>100000$ yeast cell images.

2) All reporters for these inducible systems must be designed to be uniform, e.g., in exactly the same number of copies and introduced in the same genomic locus. 
For some inducible promoters, the level of activity depends on the level of the inducer. However, given that the input-output relationships for some of the promoters investigated here are known to be highly sigmoidal ${ }^{20,21}$, we focused on the characterization of the promoters' dynamic properties, not doseresponse curves.

Here, we present:

1) a single-cell based characterization of widely used inducible promoters, identifying several noteworthy features of these promoters that experimentalists need to be aware of,

2) the extraction of key parameters from the time courses: on time lag, off time lag, induction slope and strength, and leakage (population level averages and cell-to-cell variability),

3) an analysis of the ARG3 promoter for potential use as an inducible system,

4) a demonstration of how this data enables fine experimental tuning of the timing of successive cell-cycle transition with close-to-wild-type timing, and

5) the set of budding yeast strains used here, which will be shared with other laboratories to benchmark to-be-developed inducible systems.

\section{Results}

\section{Construction of the promoter-fluorescent protein library}

In order to characterize the inducible systems in a systematic manner, we first constructed a comprehensive library of promoters driving the expression of $y E V e n u s^{22}$, a bright and fast-folding ${ }^{23}$ yellow fluorescent protein optimized for expression in yeast, fused to a constitutive degron (PEST) from the CLN2 gene, which leads to fast degradation of the protein. ${ }^{24}$ The yEVenus-PEST construct has been extensively used in the past, including as a transcriptional reporter in budding yeast. ${ }^{25,26}$

Several factors such as the genomic integration site ${ }^{27,28}$, the distance between the promoter and the gene ${ }^{29}$, and the terminator sequence ${ }^{30}$ are known to influence the expression level of the reporter gene in budding yeast. In addition, genetic constructs can be integrated with different copy numbers in the genome, resulting in different levels of expression and noise. ${ }^{31,32}$ To allow direct comparisons between the inducible promoters, we built the promoter-yEVenus circuits using the same plasmid backbone sequence and the same cloning strategy and we integrated them as single copies in the same locus (URA3) in the genome (Methods).

In the promoter-yEVenus library, we included yeast promoters that have been widely used for controlling gene expression: MET3pr ${ }^{33}, \mathrm{GAL}_{\mathrm{pr}}{ }^{34}, \mathrm{GALLpr}^{20}, \mathrm{CUP} 1 \mathrm{pr}^{35}$, and PHO5pr ${ }^{36}$. We also included the tetracycline-inducible Tet-On system, regulated by the rtTA transcription factor, which is active in the presence of tetracycline or doxycycline ${ }^{37}$ and which we call tetOpr. Lastly, we include two lightinducible systems: a promoter comprised of light-sensitive transcription factor binding sites fused to a minimal promoter ${ }^{4}$, which we refer to as LIP ("light-inducible promoter"). Another light-inducible promoter is based on the GAL1 promoter with the GAL4 activator binding sites deleted ${ }^{38}$, which we call GLIP ("GAL1pr-based light-inducible promoter"). Both of these promoters are regulated by the EL222 transcription factor ${ }^{39}$, which is active in the presence of blue light and in darkness spontaneously reverts to its inactive state.

In order to prevent transcriptional read-through, some researchers have placed a terminator upstream of the genetic circuit of interest. ${ }^{1,40-42}$ It has been suggested that in yeast, terminators themselves can function as promoters due to the presence of a hexamer motif which resembles the TATA box sequence, required for transcriptional initiation. ${ }^{43}$ However, the effect that an upstream terminator has on 
gene expression has not been determined. To test whether an upstream terminator modulates the activity of the downstream expression cassette, we used the doxycycline-inducible promoter (tetOpr) with the ADH1 terminator placed upstream of the promoter ( $t$-tetOpr).

\section{Measurements of single-cell time courses}

We measured the induction dynamics by tracking single cells by time-lapse microscopy. Cells were grown in non-inducing media overnight $(>12 \mathrm{~h}$ ), after which the promoter-yEVenus circuit was induced for 3.5 hrs, then shut off, and monitored for another 3 hrs. The period of induction corresponded to roughly 2.5 budding yeast cell cycles in glucose media, a sufficiently long time for many applications. A summary of the induction and repression conditions is given in Table 1 (detailed description of the media is given in Supplementary note 1).

\begin{tabular}{|c|c|c|}
\hline Promoter & Inducing condition & $\begin{array}{c}\text { Non-inducing or repressing } \\
\text { condition }\end{array}$ \\
\hline GAL1pr, GALLpr & Galactose & Glucose, raffinose \\
\hline LIP, GLIP & Blue light & Absence of blue light \\
\hline tetOpr, t-tetOpr & Doxycycline & Absence of doxycycline \\
\hline MET3pr & Absence of methionine & Methionine \\
\hline CUP1pr & Copper & Absence of copper \\
\hline PHO5pr & $\begin{array}{c}\text { Absence of inorganic } \\
\text { phosphate }\end{array}$ & Inorganic phosphate \\
\hline
\end{tabular}

Table 1. Inducing and non-inducing conditions used for controlling the activity of different promoters.

The strength of the systems varied 50 fold (Fig. 1). Interestingly, several promoters showed complex dynamics upon induction. The initially weak activation of PHO5pr is followed by a substantially stronger induction starting at around $\mathrm{t}=2 \mathrm{~h}$. The tetO promoters showed a substantial delay in shut-off compared to other promoters. MET3pr and GALLpr showed a decline in activity for $\mathrm{t}>1.5 \mathrm{~h}$. In addition, CUP1pr and GALLpr showed strong temporal fluctuations in promoter activity (single-cell trajectories in Fig. $1 \mathrm{I}, \mathrm{J})$. We found that a terminator placed upstream of the tetO expression cassette had no substantial effect on the expression dynamics. 
A Transcriptional reporter

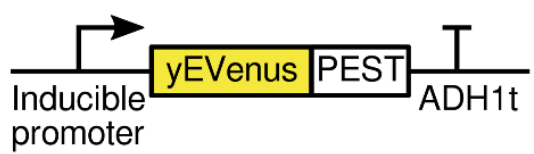

C

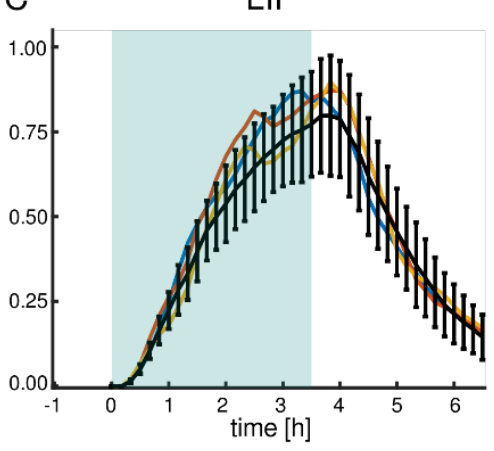

E

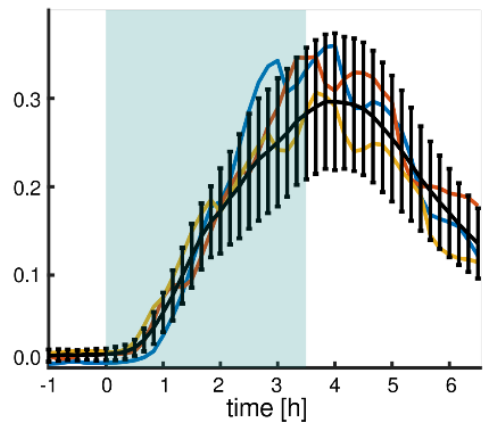

G

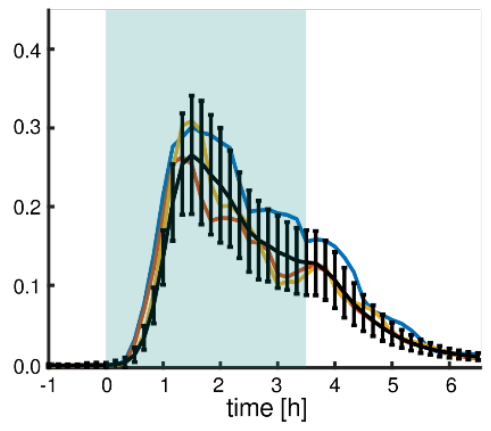

I

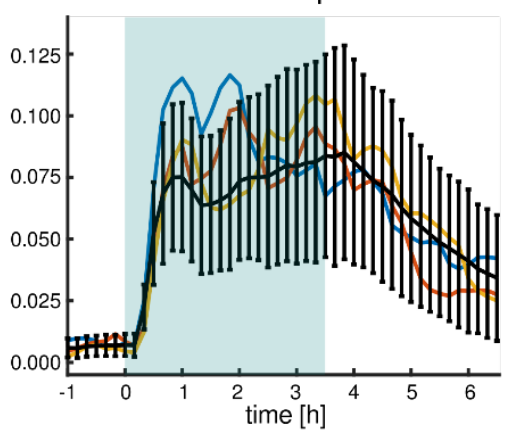

B

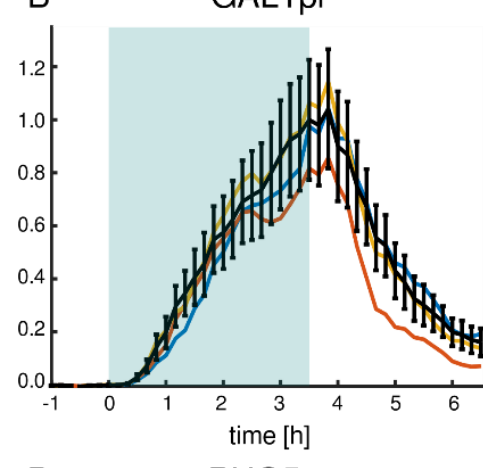

D $\quad$ PHO5pr

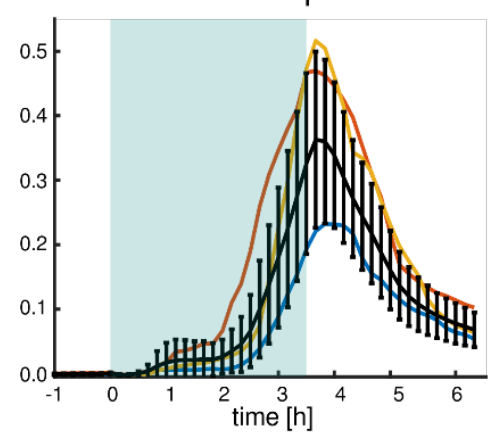

F tetOpr

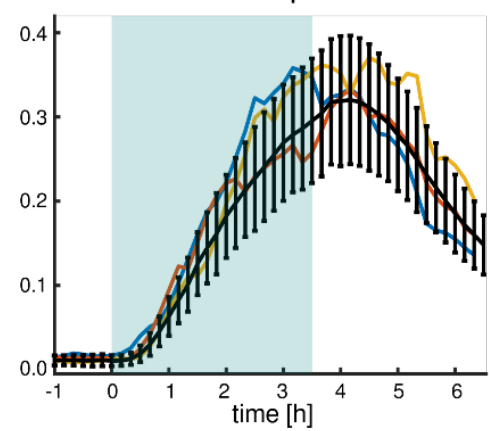

H GLIP
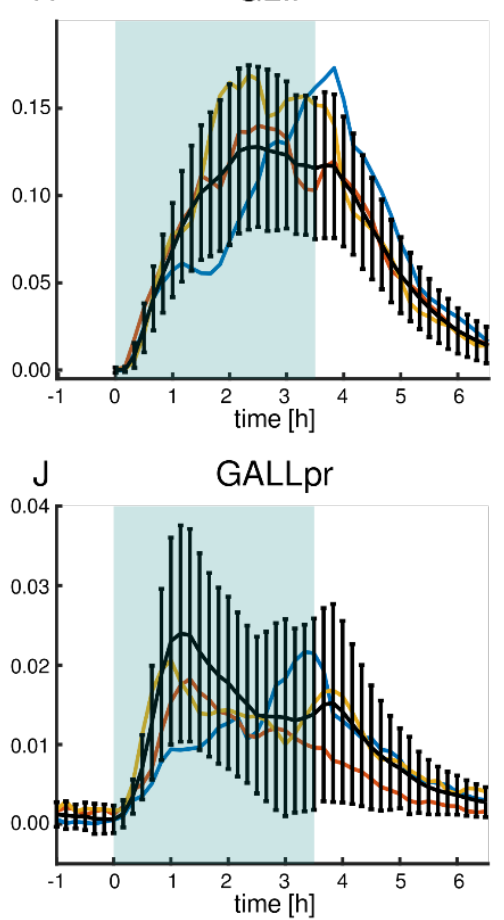

Figure 1. On and off dynamics of inducible promoter systems. A: The reporter for transcriptional activity consists of an inducible promoter and the fast-folding yellow fluorescent protein $y E V e n u s$ fused with a constitutive degron (PEST) and the ADH1 terminator. B-J: Time courses of activation and deactivation for different inducible promoters sorted in descending order by peak strength. Induction starts at $\mathrm{t}=0 \mathrm{~h}$ and finishes at $\mathrm{t}=3.5 \mathrm{~h}$. The blue background represents the induction period. Fluorescence is scaled relative to GAL1pr fluorescence at $3.5 \mathrm{~h}$. Black lines show the average of the mean cellular expression and standard deviation. Colored lines show different representative single-cell time courses. For the light-inducible promoters (LIP and GLIP), fluorescence was not measured prior to induction in order to avoid possible promoter activation by the light source used for fluorescent protein excitation. 


\section{Characterization of the kinetics, cell-to-cell variability, and effect on growth}

We fitted the expression data to a coarse-grained model (Fig. 2 A) to extract quantitative parameters from the single-cell time courses (Fig. 2 B-G). Variations of this model have been used previously for characterizing gene expression kinetics. ${ }^{26,31}$ In the model, the basal (non-induced) expression is controlled by $b$. Promoter activity upon inducer addition is determined by an initial lag $t$-on between the moment the signal arrives and when induction starts, the slope $i$ of the subsequent induction, and the peak strength of the induction. The period after the inducer is removed is characterized by the lag in time $t$-off between when the signal is removed and when induction starts decreasing. Two more parameters are the degradation constant of the unfolded and folded proteins $d$ which, as expected since the same protein is expressed in all cases, was very similar for different promoters, and the folding / maturation time for the fluorescent protein $f$, which has been measured in a number of studies to be $17.6 \mathrm{~min}$.

\begin{tabular}{|c|c|c|c|}
\hline$A$ & 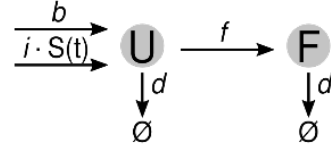 & $\begin{array}{l}\text { U - Unfolded protein } \\
\text { F - Folded protein } \\
\text { S(t) - Input }\end{array}$ & $\begin{array}{l}\mathrm{dU} / \mathrm{dt}=b+i \cdot \mathrm{S}(\mathrm{t})-(d+f) \cdot \mathrm{U} \\
\mathrm{dF} / \mathrm{dt}=f \cdot \mathrm{U}-d \cdot \mathrm{F} \\
\mathrm{S}(\mathrm{t})=\mathrm{H}\left(\mathrm{t}-t_{o n}\right) \cdot \mathrm{H}\left(3.5 \mathrm{~h}+t_{o f f}-\mathrm{t}\right)\end{array}$ \\
\hline
\end{tabular}
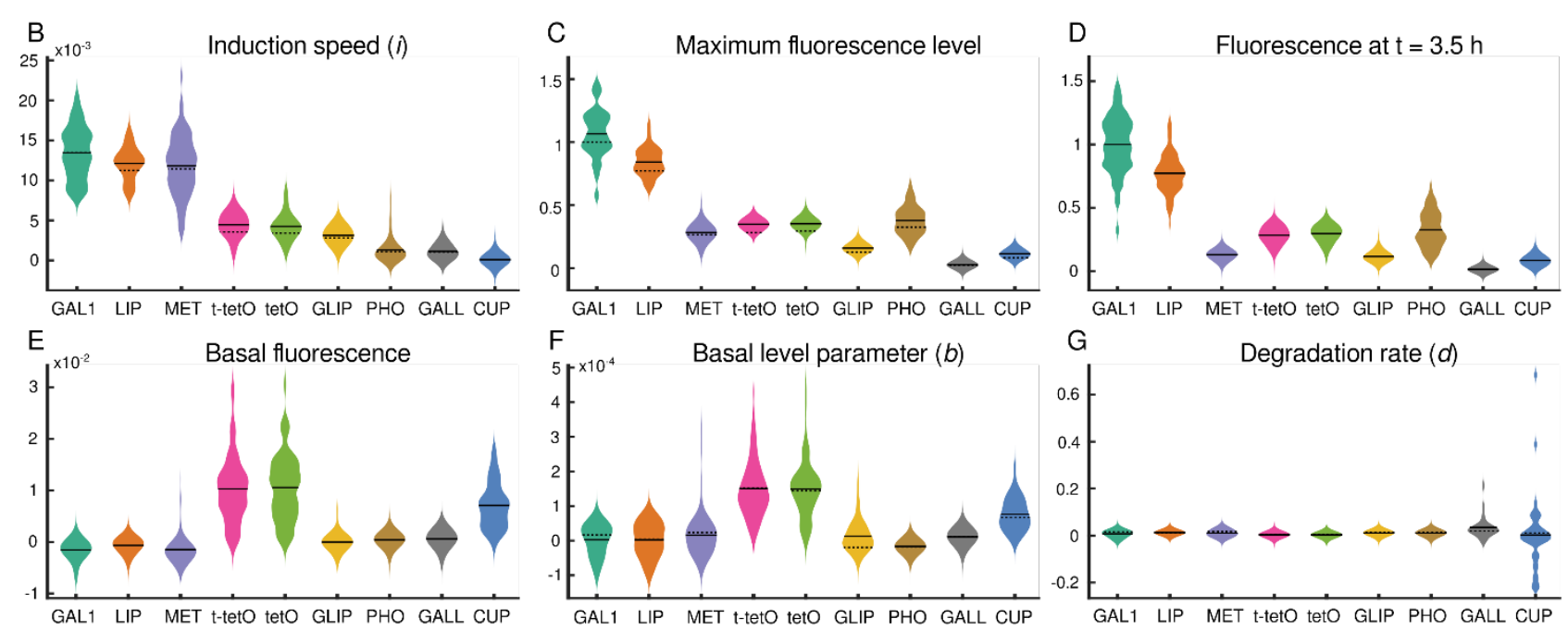

Figure 2. Single-cell-level characteristics of the inducible systems. A: Model of gene expression used to extract the quantitative parameters describing the inducible promoters. $\mathrm{H}(\mathrm{t})$ is the Heaviside step function, 0 for $\mathrm{t}<0$ and 1 for $\mathrm{t}>=0$. B: Speed of induction i. C: Maximum fluorescence levels normalized by mean GAL1pr levels at $3.5 \mathrm{~h}$. D: Fluorescence levels at the end of the induction period. E: Basal fluorescence levels. F: Basal activity parameter $b$. G: Degradation rate $d$. B, F, D: Violin plots show distributions of parameters estimated by fitting fluorescence levels in single cells. Black solid lines show the mean of the distribution. When applicable, fitting was performed on promoter activities which were first averaged over the population of cells, and these values are shown by dashed black lines. "pr" in the promoter names omitted for brevity.

The characterized promoters' initial speed $i$ spanned a 10 fold range, with GAL1pr being the strongest and CUP1pr the weakest tested promoter (Fig. 2 B). Most of the promoters showed no measurable activity in the off state, that is, no leakiness at the sensitivity of fluorescence microscopy (Fig. $2 \mathrm{E}$ ). (We measured basal fluorescence levels by flow cytometry for GAL1pr, GALLpr, MET3pr, LIP, and GLIP as well and saw no basal fluorescence above wild-type autofluorescence levels.) Exceptions are CUP1pr and both versions of the tetOpr system, which showed considerable levels of expression (approx. $1 \%$ of peak GAL1pr expression) in the absence of the inducing signals. For MET3pr, we observed a fraction of the population of cells that showed non-zero activity in the off state, which may be relevant when considering their use when tight regulation is needed. Estimated degradation rates are similar for all with the exception of GALLpr, which showed substantial variations in degradation rates due to the large 
temporal fluctuations during the induction period introducing large variability in the fitted estimates (single-cell trajectories of cells shown in Fig. 1).

The relative leakiness, strength of induction, and maximal levels of induction are summarized below:

Leakiness:

tetOpr $\approx$ t-tetOpr $>$ CUP1pr $>$ GALLpr $\approx$ PHO5pr $\approx$ GLIP $\approx$ LIP $\approx$ MET3pr $\approx$ GAL1pr

Strenght of induction:

GAL1pr $>$ LIP $>$ MET3pr $>$ tetOpr $\approx$ t-tetOpr $>$ GLIP $>$ PHO5pr $>$ GALLpr $>$ CUP1pr

Max. level:

GAL1pr $>$ LIP $>>$ PHO5pr $>$ tetOpr $\approx$ t-tetOpr $>$ MET3pr $>$ GLIP $>$ CUP1pr $>$ GALLpr

Expression systems may interfere with growth due to the toxicity of the inducer or a metabolic burden $^{18}$, for example, a suboptimal carbon source. To benchmark promoters in terms of cell growth, we measured the doubling times of the area of the colony during the last hour of the induction $(2.5 \mathrm{~h}<\mathrm{t}<3.5$ h) (Fig. $3 \mathrm{~A}$ ). The induction of the light-inducible promoters was surprisingly harmful, given that we utilized the diascopic white light of the microscope at $80 \%$ strength. We repeated our LIP measurements with $20 \%$ light intensity and observed $25 \%$ less induction but a more healthy 90 min area doubling time. We include the 20\% light intensity results in Fig. 5 but proceed with the results for the stronger $80 \%$ induction in the following.

Doubling times:

tetOpr $\approx$ t-tetOpr $<$ MET3pr $<$ PHO5pr $<$ CUP1pr $<$ GLIP $<$ GALLpr $<$ GAL1pr $<$ LIP

The lag times turned out to be particularly sensitive to temporal fluctuations in the single-cell time courses. Given these strong fluctuations for some of the promoters, we extracted the delay upon activation and upon deactivation of the promoters for the averaged time course data (Fig. 3 B and C)

Doubling-time, ON delay and OFF delay are summarized below:

t-on: GLIP $<$ CUP1pr $<$ GALLpr $<$ LIP $<$ PHO5pr $<$ GAL1pr $<$ tetOpr $\approx$ t-tetOpr $<$ MET3pr

t-off. GAL1pr $<$ GALL $<$ GLIP $<$ CUP1pr $<$ tetO $<$ LIP $<$ PHO5pr $<$ MET3pr 
A

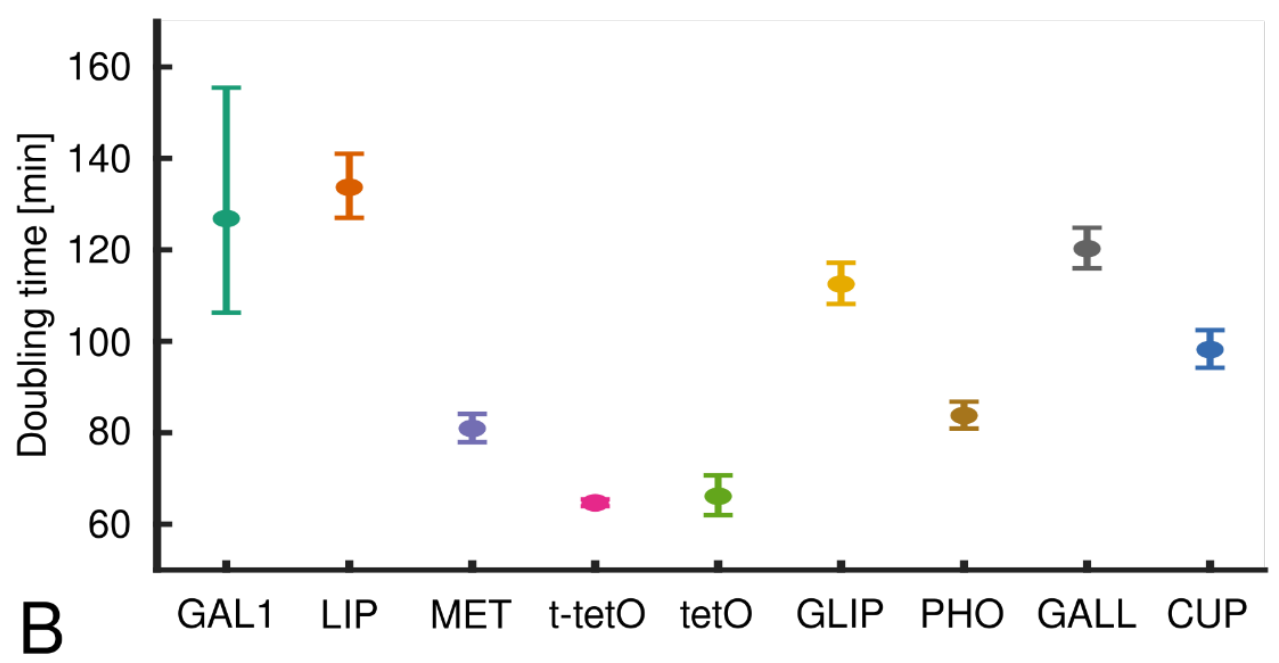

Figure 3. Growth burden, time delay after activation ( $t$-on) and after deactivation ( $t$-off) for different promoters, "pr" in the promoter names omitted for brevity. Standard errors of the mean for $t$-on and $t$-off delays were estimated by bootstrapping single-cell expression values and fitting 100 averaged time courses to the model.

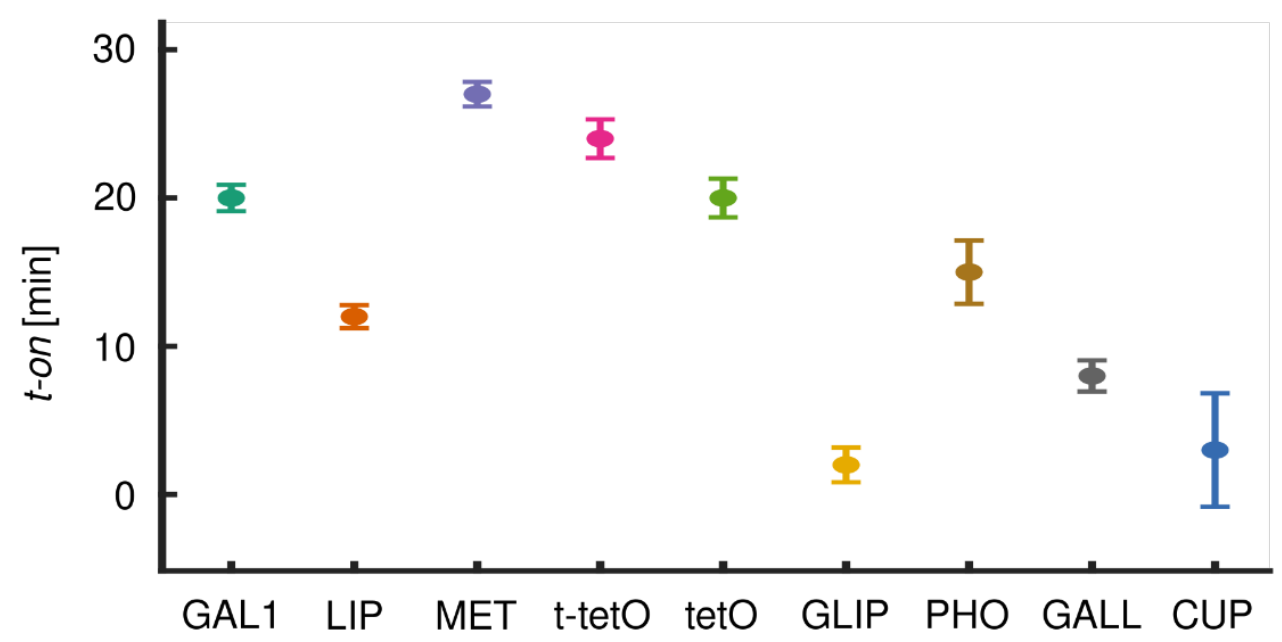

C

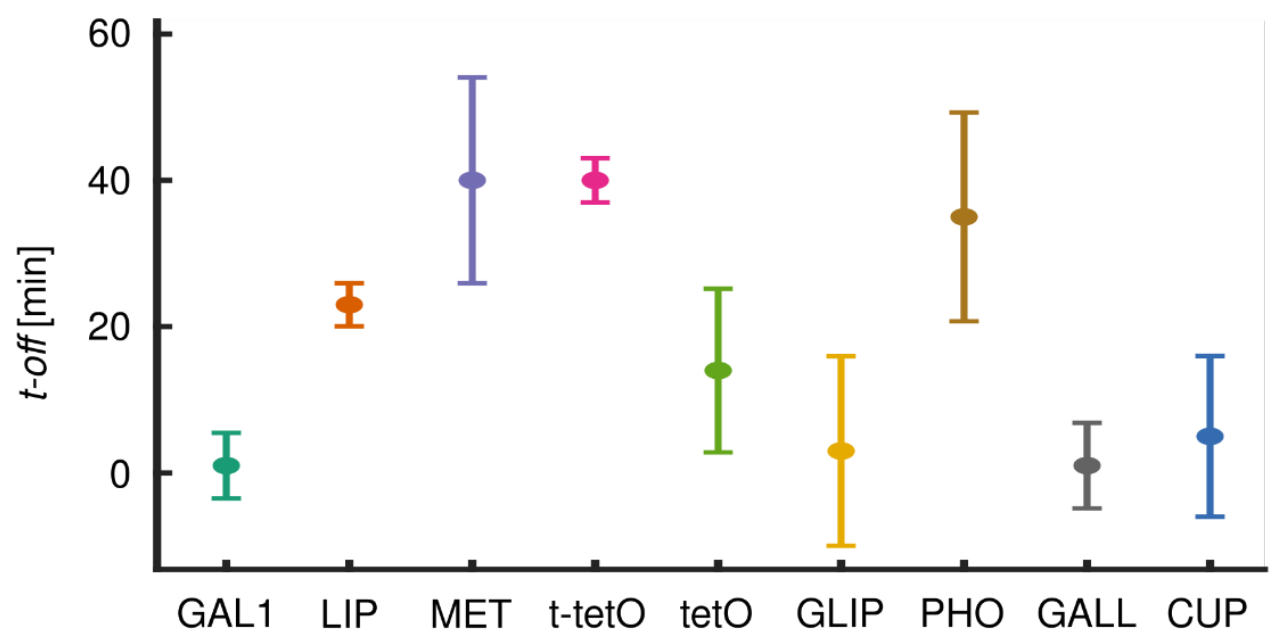


PHO5pr, although switched off within $20 \mathrm{~min}$, causes the cells to arrest, presumably due to the absence of inorganic phosphate, which is essential. Cells do not show any cell-cycle related activity even $7 \mathrm{~h}$ after the inorganic phosphate is supplied back to the media (data not shown), which imposes a severe limitation on applications using PHO5pr as an inducible promoter.

Even within a population of genetically identical cells, the responsiveness of a genetic circuit can vary, which is in many cases an undesirable property for bioengineering applications. The relationship between mean and standard deviation levels can be complex. ${ }^{44}{ }^{45}$ To determine this relationship for the expression levels of the inducible promoters, we calculated the coefficient of variation for the last timepoint $(\mathrm{t}=3.5 \mathrm{~h})$ of induction in the time course experiment (Fig. 4).

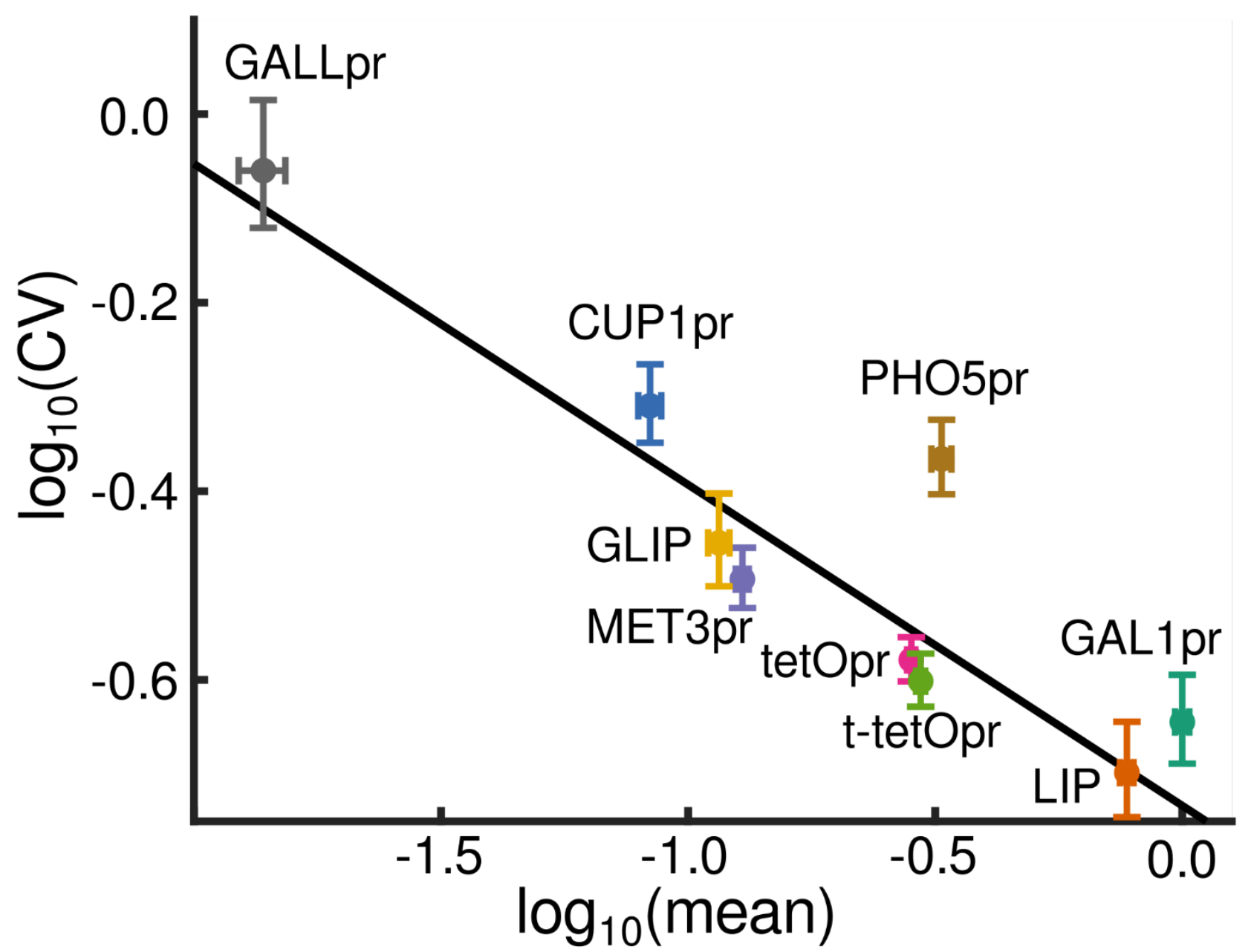

Figure 4. Log of noise (CV) versus mean of inducible promoter expression levels are inversely correlated. Noise is calculated as the coefficient of variation for the population of cells at the last timepoint of induction in the time course experiment $(t=3.5 \mathrm{~h})$. All fluorescence values were normalized by dividing the mean at $\mathrm{t}=3.5 \mathrm{~h}$ by the mean for GAL1pr as shown in Fig. 1. The least squares regression was computed with PHO5pr excluded (slope $=-0.34$, 95\% confidence interval: $[-0.4344,-0.2463])$.

As expected ${ }^{46}$, the strength of the noise decreased with the increase in the mean expression level. In the log-log plot, a least squares regression line fit the data points neatly (slope $=-0.34$ ) when we removed the point for PHO5pr, which showed a high level of noise for its mean expression level (Fig. 4).

For many applications, the strength of the induction, the effects on growth rates, and the leakiness are likely to be important. Fig. 5 represents the striking trade-offs that exist between these properties for existing inducible promoter systems. 


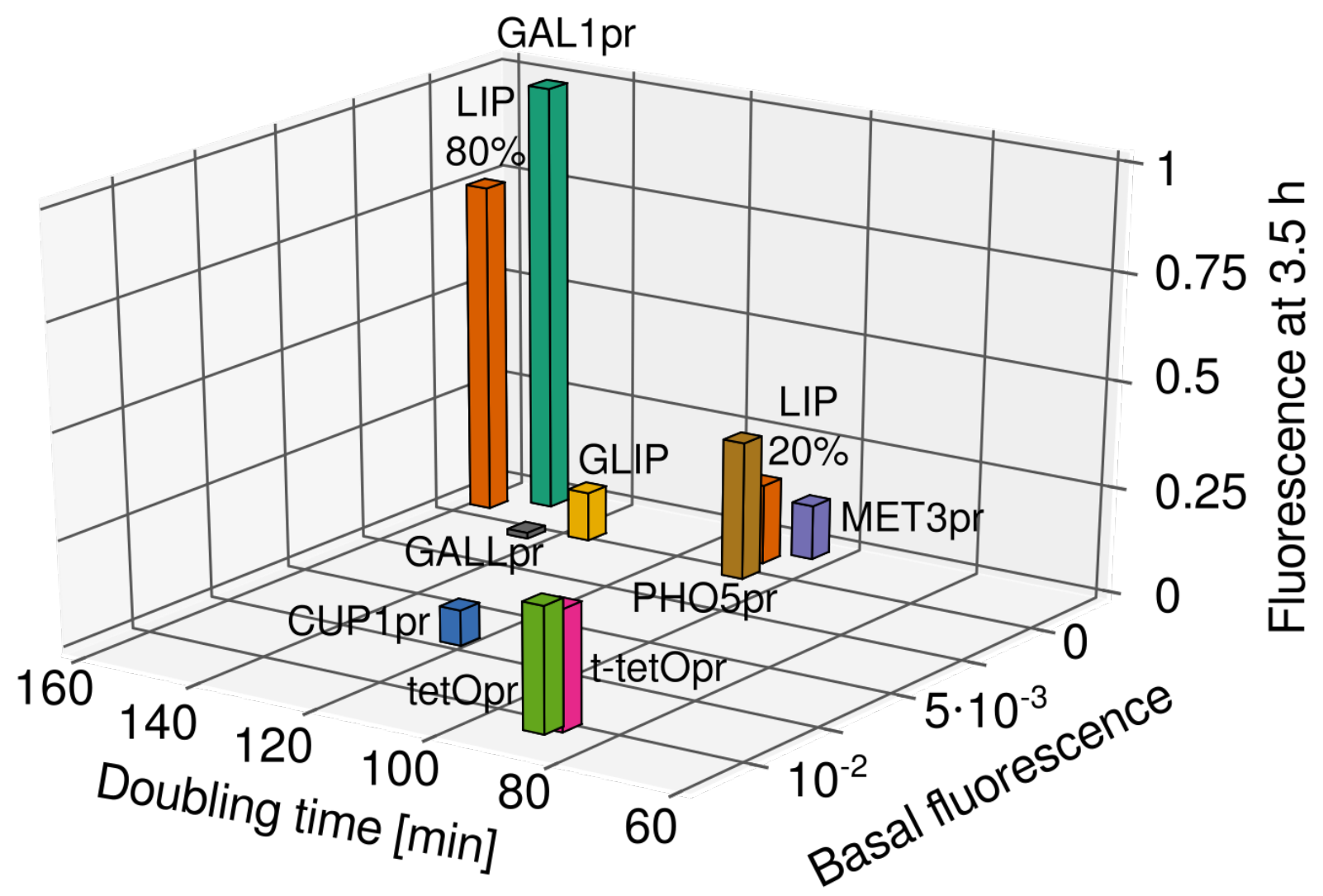

Figure 5. Multidimensional benchmarking of promoters illustrates the trade-offs for inducible systems. The underlying data is the same as in Figs. 2 and 3. All fluorescence values are relative to GAL1pr levels at $3.5 \mathrm{~h}$.

\section{Characterization of the arginine-responsive promoter ARG3pr}

We decided to expand the previous analysis by an additional system, which has previously not been characterized for potential use as an inducible system. ARG3 is a part of the arginine-synthesis pathway in budding yeast and ARG3 mRNA was identified as strongly upregulated under amino acid starvation ${ }^{47}$. ARG3pr is known to be repressed by arginine and can be additionally regulated by different amino acids or chemicals used as a nitrogen source such as methionine ${ }^{48,49}$. During the initial test, we grew yeast cells transformed with a single copy of an ARG3pr-yEVenus construct in media containing 10x concentrations of arginine, methionine, both, or none. We found that ARG3pr is activated more strongly in both the absence of arginine or the presence methionine $(-A$ OR $+M)$. However, its basal level of activity in the presence of arginine and absence of methionine was high (Fig. $6 \mathrm{~A}$ ).

To characterize the dynamics of switching between the off state (in $+M+A$ media) to the on state (in $+\mathrm{M}-\mathrm{A}$ media), we analyzed the ARG3pr-yEVenus expression time courses. Although at the population level, the ARG3 promoter showed stable changes in activity in the presence of different media, single-cell trajectories showed strong oscillations with the cell-cycle period (Fig. $6 \mathrm{C}$ ), favoring this promoter's use in bulk culture. 
A

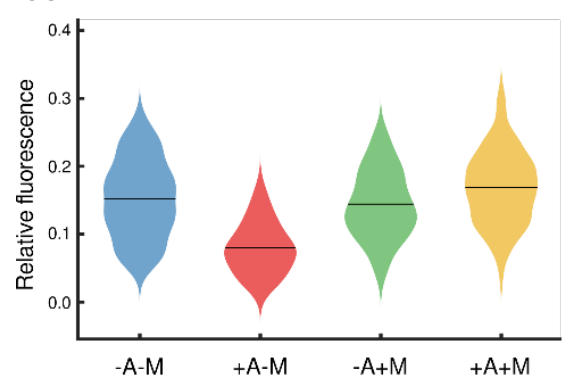

B

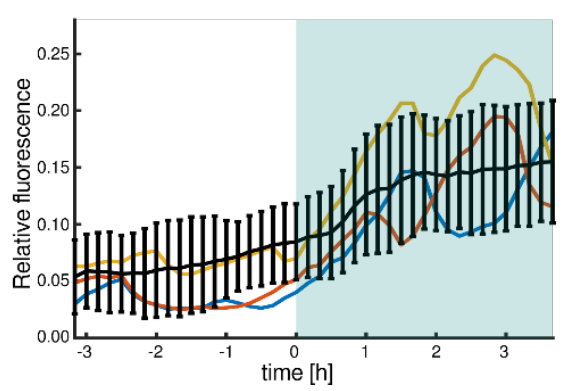

C

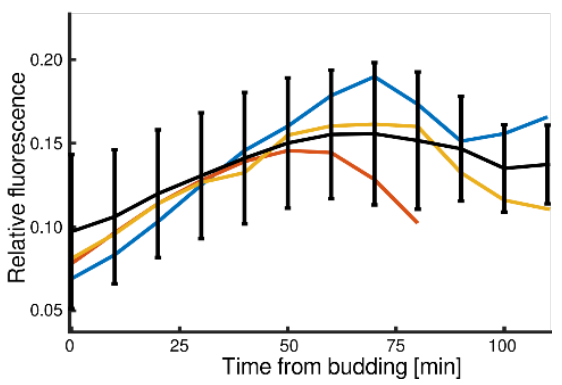

Figure 6. Dynamic properties of the ARG3 promoter. A: Mean activity of ARG3pr in different media. $+\mathrm{A}$ or $+\mathrm{M}$ denotes 10x concentrations of arginine or methionine, respectively, and -A and -M denote the lack of arginine or methionine in the media. B: Time courses of ARG3-yEVenus activity in media lacking methionine. The switch from $+\mathrm{A}$ to $-\mathrm{A}$ occurred at $0 \mathrm{~h}$. Black line represents the average of the cells' fluorescence levels while colored lines represent examples of single-cell fluorescence time courses. C: Alignment of different single-cell trajectories around the budding time show that ARG3pr is likely cell-cycle regulated. In all panels, fluorescence is normalized with respect to GAL1pr fluorescence at the $3.5 \mathrm{~h}$ time point.

ARG3pr responds quickly to the removal of arginine in the media, suggesting that it could be used as a biosensor for arginine (Fig. $6 \mathrm{~B}$ ). In addition, given that ARG3pr is regulated by methionine in the opposite way from MET3pr, which is suppressed by methionine, ARG3pr can be used in a setting where inverted control of two circuits is needed.

\section{Experimentally tuning the time between Start and mitosis}

One of the goals of synthetic biology is to engineer complex artificial cellular behaviors. This often requires multiple inducible promoters to be controlled simultaneously with high temporal precision. A scenario where such precision is necessary is in controlling inherently dynamic systems such as the cell cycle. Here, we control the lag between cell cycle Start and mitosis by independently inducing the expression of Start and M-phase cyclins in succession.

Cyclins are a group of regulatory proteins, which, together with the cyclin-dependent kinase Cdk1 control the processes required for cell-cycle initiation, progression, and exit. ${ }^{50} \mathrm{G} 1$ cyclin (CLN3) or G1/S cyclins (CLN1,2) trigger entry into the cell cycle, while M phase cyclins (CLB1, CLB2) trigger mitosis. ${ }^{50}$

In order to control entry into the cell cycle, we used the well-known MET3pr-CLN2 construct, which controls cell cycle Start in a strain in which all other Start cyclins have been deleted (cln1-3A). To tune the expression of the major mitotic cyclin CLB2, whose rate of expression is known to be limiting for the speed of mitosis ${ }^{51,52}$, we put an undegradable version of this cyclin (CLB2kd) ${ }^{53}$ downstream of LIP. We chose LIP among other tested promoters because its induction strength is comparable to the strongest promoter tested (GAL1pr) but does not require raffinose or galactose as a carbon source, which are less optimal for growth than glucose. In addition, LIP induction can be modulated by varying the light intensity ${ }^{4,38}$. LIP-CLB2kd is solely responsible for mitotic entry in a strain in which both mitotic cyclins were deleted $(\mathrm{clb1}, 2 \Delta)$. This strain is kept viable by a GALLpr-CLB2 construct in galactose medium prior to the experiments. ${ }^{54}$ Cells lacking all G1 and G1/S cyclins are arrested in the G1 phase, while cells lacking CLB1 and CLB2 are arrested prior to M phase. 
A

$\begin{array}{cccccc}\text { Growth } & \begin{array}{c}\text { Cell cycle } \\ \text { synchronization }\end{array} & \text { Re-arrest } & \begin{array}{c}\text { GALLpr- } \\ \text { CLB2 off }\end{array} & \begin{array}{c}\text { MET3pr- } \\ \text { CLN2 on } \\ \text { variable times }\end{array} & \begin{array}{c}\text { LIP-CLB2kd } \\ \text { on } \\ \text { until end }\end{array} \\ \text { G-Met } & 2 \mathrm{~h} & 50 \mathrm{~min} & 20 \mathrm{~min} & 1 \mathrm{~h} & \end{array}$
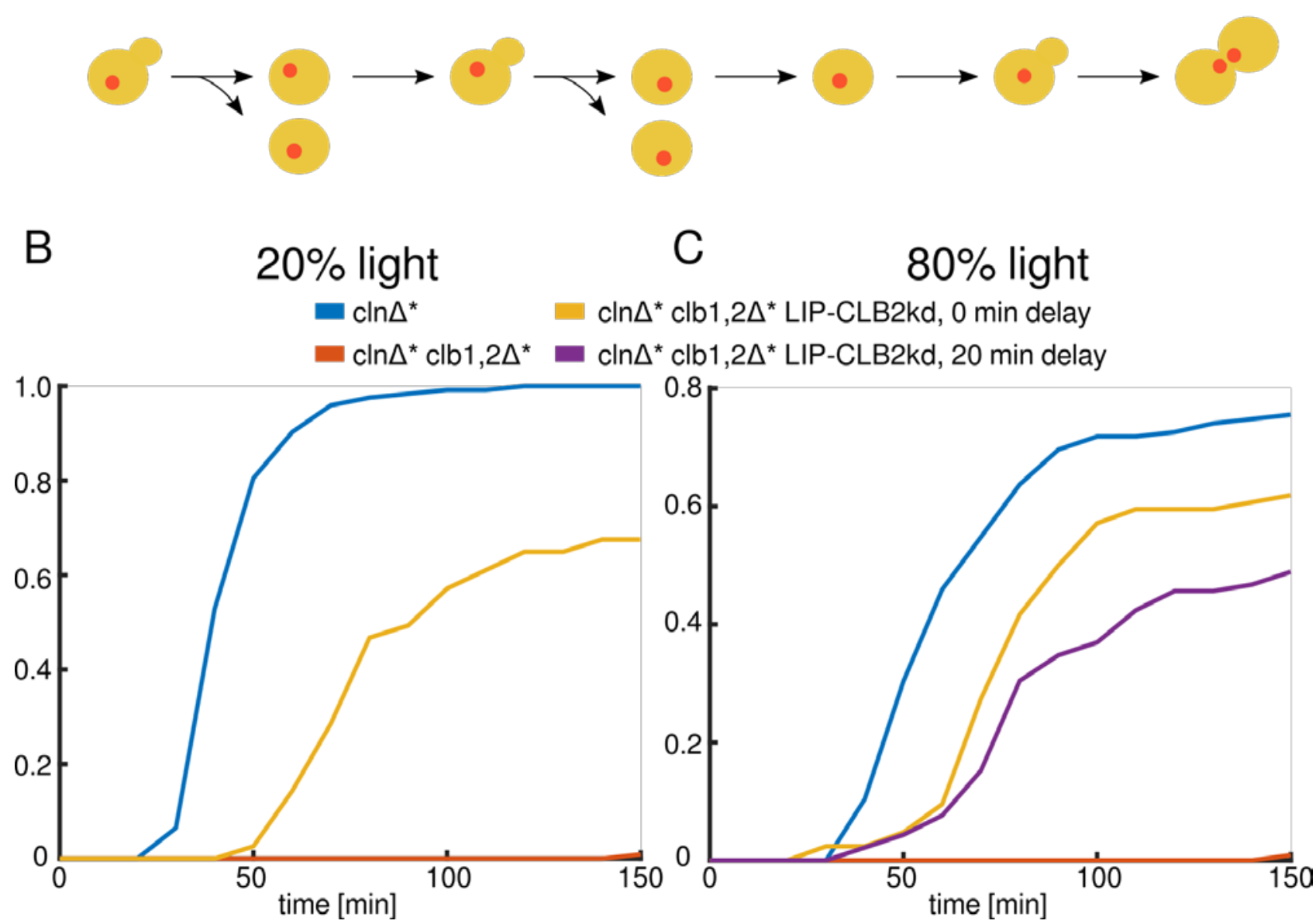

Figure 7. Independent triggering of cell cycle Start and mitosis to simulate wild-type timing. A: Illustration of the protocol. B: Budding-to-anaphase duration with $20 \%$ diascopic light intensity. C: Budding-to-anaphase duration with $80 \%$ light intensity. $\operatorname{cln} \Delta^{*}$ denotes $\operatorname{cln} \Delta$ MET-CLN2pr while clb1,2 $\Delta^{*}$ denotes clb1,2 $\Delta$ GALLpr-CLB2. The same experiment with control $\operatorname{cln} \Delta^{*} \mathrm{clb} 1,2 \Delta^{*}$ cells (without the LIP-CLB2kd construct) is shown in panels B and C.

Before inducing LIP-CLB2kd, we ran cells through a sequence of media switches designed to deplete the Clb2 protein generated from GALLpr-CLB2. We call these steps the Clb depletion protocol ${ }^{55}$ (Fig. 7 A). After growing cells for several hours in G-M media, where both MET3pr-CLN2 and GALLprCLB2 constructs were expressed, we synchronized the population by switching the media to G+M (in which cells arrest in G1) for $2 \mathrm{~h}$. Then, the medium was switched back to G-M for 50 min, and cells restarted the cell cycle. In this way, we ensured that in any subsequent steps, cells were in a similar cell cycle stage. We then turned the GALLpr-CLB2 construct off while cells were at the end of this cycle so that the inhibitors of the Clb2 protein that are expressed in anaphase and later degrade the leftover Clb2 from the GALLpr-CLB2 construct. Lastly, we released cells from the arrest by switching the media from $+\mathrm{M}$ to $-\mathrm{M}$ and began the main experiment by turning on the light source, which activated the LIP-CLB2kd construct. 
We varied LIP-CLB2kd expression by changing the level of light that cells were exposed to and by changing the delay between the -M pulse, which triggered entry into the cell cycle, and the light pulse, which triggered mitotic entry. To monitor the dynamics of the cell cycle, we included the fluorescently labeled HTB2-mCherry ${ }^{56}$ construct in our strains, which marked the position of the nucleus throughout the whole cell cycle. As a proxy for cell-cycle duration, we measured the time from bud appearance to the separation of the fluorescently labeled nuclei in anaphase.

First, we applied the microscope's LED diascopic light set to $20 \%$ of the maximal intensity (Fig. 7 B). Around $60 \%$ of cells with the LIP-CLB2kd construct that budded successfully finished mitosis. However, their speed was substantially slower than cells with wild-type CLB1,2. Nevertheless, the effect was due to timely expression from the LIP-CLB2kd construct since residual Clb2 from the GALLpr-CLB2 construct was not enough to drive cells through mitosis; this was verified by detecting no nuclear divisions in cells without the LIP-CLB2kd construct (Fig. 7 B and C). The Clb-depletion protocol indeed removed Clb proteins sufficiently.

In order to observe the effects of stronger LIP-CLB2kd induction, we applied light with $80 \%$ of the maximal intensity (Fig. 7 C). This decreased the time from bud emergence to nuclear separation with the proportion of cells that finish mitosis remaining relatively unaffected. On the other hand, we could modulate the dynamics of mitosis progression by delaying the LIP-CLB2kd pulse relative to the MET3prCLN2 pulse. However, the proportion of CLB wild-type cells that finished mitosis in the presence of $80 \%$ light reduced substantially, from around $100 \%$ in the presence of $20 \%$ light to around $75 \%$ in the presence of $80 \%$ light. This suggests that higher intensity of light is toxic for cycling cells and that presumably different underlying effects cause cells with the LIP-CLB2kd construct to not finish mitosis in these two cases, inappropriate rate or timing of the CLB2kd pulse in one case and light toxicity in the other case.

\section{Discussion}

Quantitative characterizations of inducible systems are needed to guide experimental designs. Here, we systematically benchmarked the characteristics of inducible systems in budding yeast.

Different experiments might require promoters with different maximal levels of induction, or may tolerate different maximal levels of leakiness or growth burden. To show how the multidimensional characterization presented here highlights the drawbacks of the widely used inducible promoters for budding yeast, we plotted the relationship between maximal levels of induction, leakiness, and growth burden (Fig. 5). Strong promoters, such as LIP and GAL1pr are associated with slow cellular growth likely due to phototoxicity and a suboptimal carbon source, respectively. The weaker promoters tetOpr, MET3pr, GALLpr, and CUP1pr, either show substantial levels of leakiness (tetOpr) or show fluctuations in time (MET3pr, GALLpr, and CUP1pr).

We showed that the maximal level of induction of the tested promoters span a $>50$ fold range, suggesting that the library described here is diverse enough for different choices of inducible systems in the future. With kinetic and steady-state parameters taken together, none of the tested promoters performed optimally, emphasizing the need for the multidimensional characterization performed here and the need for the development of novel tools for the precise dynamic control of cellular processes.

The comparatively little explored ARG3 promoter showed an interesting OR gate behavior as well as the opposite activation with respect to methionine levels than MET3pr.

Lastly, we showed that with two relatively fast promoters, we could simulate the succession of cell cycle Start and mitosis with nearly wild-type timing. 


\section{Methods}

\section{Plasmid library construction}

All plasmids were constructed and propagated using E. Coli DH5 $\alpha$ strain. DNA digestion and ligation were performed using restriction endonucleases and T4 DNA ligase from New England Biolabs (USA). Promoter-yEVenus Library was constructed by cloning different promoter sequences upstream of the yEVenus ORF using PacI and BamHI restriction enzymes. All PCRs were performed with Phusion Polymerase (New England Biolabs, USA). All constructs were verified by Sanger sequencing (Microsynth AG, Switzerland). Summary and details of the construction of plasmids used in this study are given in the Supplementary Table 1.

\section{Strain construction}

Wild-type haploid W303 budding yeast strains (MATa ade2-1 leu2-3 ura3-1 trp1-1 his3-11,15 can1-100) were transformed with plasmids with the inducible promoter-yEVenus constructs by digesting the plasmid with StuI endonuclease inside the URA3 gene. Transformations were performed using the standard lithium acetate method ${ }^{57}$ and selection of transformed strains was done using the appropriate dropout agar plates. For promoters that are induced by synthetic transcription factor activation (lightinducible and doxycycline-inducible promoters), constructs encoding transcription factors were transformed in a strain of the opposite mating type from the strain containing the promoter-yEVenus construct and the transcription factor plasmids were integrated into the HIS3 locus. The two strains were then crossed and the resulting progeny that contained both transcription factor and promoter-yEVenus constructs were selected and used in further experiments. Plasmid integration and construct activity were verified by fluorescence microscopy after the appropriate induction of the constructs. Strains that showed fluorescence were screened for single-copy integrations using the polymerase chain reaction (PCR) with primer sets that alloweded one or several copies of the construct in the genome to be distinguished (Supplementary Note 3). Summary and details of the strain construction used in this study are given in the Supplementary Table 2.

\section{Media and growth conditions}

Cells were grown in CellASIC ONIX plate for haploid yeast cells in media controlled by the ONIX2 microfluidics system (Merck, Germany). Details about the composition of the media used for different promoter induction experiments are given in the Supplementary Note 1.

For experiments with light-induced CLB2kd, cells were first grown in G-M media from a single cell into a colony for 8-12 h. After that, to ensure that no left-over of Clb2 would affect the cell cycle in which the LIP-CLB2kd construct was induced, the Clb-depletion protocol ${ }^{55}$ was applied as described in the main text.

\section{Microscopy}

Images were recorded using a Nikon Ti2-E microscope equipped with a 60x objective and a Hamamatsu Orca-Flash 4.0 camera. The microscope was operated using NIS-Elements software and the objective's axial position was controlled by Nikon Perfect Focus System. To prevent photobleaching, images were taken every $10 \mathrm{~min}$ with $100 \mathrm{~ms}$ exposure time.

\section{Image analysis}


Image analysis was performed using YeaZ, a Python-based tool for yeast cell segmentation ${ }^{19}$. First, the boundaries of cells were determined in phase-contrast images. The levels of fluorescence for each cell were calculated as an average of the pixel intensities in the yellow fluorescence channel for pixels that were within the cell boundaries. For further analyses, we subtracted the autofluorescence of unlabeled wild-type cells from the fluorescence values.

\section{Data analysis and modeling of gene expression}

For the induction kinetics, we ignored the complex dynamics observed for some of the inducible promoters by using the first 9 frames $(1.5 \mathrm{~h})$ after addition of the inducer for fitting the model and estimating the strength of induction and time delay upon induction. For CUP1pr, however, we used only the first 5 frames because its expression level start to drop thereafter. Similarly, we used the first 9 frames after the removal of inducer for extracting the fluorescent protein degradation rate and time delay upon inducer removal. Since the model parameters could not be fitted simultaneously, we used yEVenus maturation time of $0.0394 \mathrm{~min}^{-1}$ (a value which corresponds to $17.6 \mathrm{~min}$ half-life) which has been determined for the yEVenus protein used in the same organism and with the same experimental setup. ${ }^{26}$ The model fits were obtained by minimizing the sum of squared residuals using the lsqcurvefit function in Matlab 2019a.

For making violin plots, we used the bandwidth that corresponds to the value of $1.06 \sqrt[\frac{s t d}{\sqrt[5]{n}}]{\text {, as }}$ suggested in ref..$^{58}$ (std - the standard deviation, $\mathrm{n}$ - the number of elements in the set).

\section{Author contributions}

VG performed microscopy experiments. AS performed flow cytometry experiments. VG and AS analyzed the data. VG performed modelling and analyzed the results. VG created genetic constructs and strains. VG and SJR wrote the manuscript. SJR supervised the work.

\section{Acknowledgment}

We thank Dr. Enrico Tenaglia for help with the experiments and media preparation, Dr. Evan Olson and Shiyu Cheng for help with media preparation, Prof. Mustafa Khammash and Dr. Dirk Benzinger for providing plasmids and giving us technical advice for optogenetics measurements, Prof. José L. Avalos and Dr. Evan Zhao for providing plasmids, and Prof. Attila Becskei for providing plasmids.

\section{Competing interests}

The authors declare no competing interests.

\section{Data availability}

Data, plasmids, and strains used in the study are available upon request.

\section{References}

1. Becskei, A. Positive feedback in eukaryotic gene networks: cell differentiation by graded to binary response conversion. EMBO J. 20, 2528-2535 (2001).

2. Elowitz, M. B. Stochastic Gene Expression in a Single Cell. Science 297, 1183-1186 (2002). 
3. Rahi, S. J. et al. Oscillatory stimuli differentiate adapting circuit topologies. Nat. Methods 14, 10101016 (2017).

4. Zhao, E. M. et al. Optogenetic regulation of engineered cellular metabolism for microbial chemical production. Nature 555, 683-687 (2018).

5. DeJong, J. M. et al. Genetic engineering of taxol biosynthetic genes inSaccharomyces cerevisiae. Biotechnol. Bioeng. 93, 212-224 (2006).

6. Ro, D.-K. et al. Production of the antimalarial drug precursor artemisinic acid in engineered yeast. Nature 440, 940-943 (2006).

7. Elowitz, M. B. \& Leibler, S. A synthetic oscillatory network of transcriptional regulators. Nature 403, 335-338 (2000).

8. Gardner, T. S., Cantor, C. R. \& Collins, J. J. Construction of a genetic toggle switch in Escherichia coli. Nature 403, 339-342 (2000).

9. Lindstrom, D. L. \& Gottschling, D. E. The Mother Enrichment Program: A Genetic System for Facile Replicative Life Span Analysis in Saccharomyces cerevisiae. Genetics 183, 413-422 (2009).

10. Blount, B. A., Weenink, T. \& Ellis, T. Construction of synthetic regulatory networks in yeast. FEBS Lett. 586, 2112-2121 (2012).

11. Morawska, M. \& Ulrich, H. D. An expanded tool kit for the auxin-inducible degron system in budding yeast: A tool kit for the AID system. Yeast 30, 341-351 (2013).

12. Portela, R. M. C. et al. Synthetic Core Promoters as Universal Parts for Fine-Tuning Expression in Different Yeast Species. ACS Synth. Biol. 6, 471-484 (2017).

13. Hong, K.-K. \& Nielsen, J. Metabolic engineering of Saccharomyces cerevisiae: a key cell factory platform for future biorefineries. Cell. Mol. Life Sci. 69, 2671-2690 (2012).

14. Da Silva, N. A. \& Srikrishnan, S. Introduction and expression of genes for metabolic engineering applications in Saccharomyces cerevisiae. FEMS Yeast Res. 12, 197-214 (2012).

15. Belli, G. An activator/repressor dual system allows tight tetracycline-regulated gene expression in budding yeast [published erratum appears in Nucleic Acids Res 1998 Apr 1;26(7):following 1855]. Nucleic Acids Res. 26, 942-947 (1998).

16. Shimizu-Sato, S., Huq, E., Tepperman, J. M. \& Quail, P. H. A light-switchable gene promoter system. Nat. Biotechnol. 20, 1041-1044 (2002).

17. Olson, E. J. \& Tabor, J. J. Optogenetic characterization methods overcome key challenges in synthetic and systems biology. Nat. Chem. Biol. 10, 502-511 (2014).

18. Ottoz, D. S. M., Rudolf, F. \& Stelling, J. Inducible, tightly regulated and growth conditionindependent transcription factor in Saccharomyces cerevisiae. Nucleic Acids Res. 42, e130-e130 (2014).

19. Dietler, N. et al. YeaZ: A convolutional neural network for highly accurate, label-free segmentation of yeast microscopy images. http://biorxiv.org/lookup/doi/10.1101/2020.05.11.082594 (2020) doi:10.1101/2020.05.11.082594.

20. Mumberg, D., Muller, R. \& Funk, M. Regulatable promoters of Saccharomyces cerevisiae: comparison of transcriptional activity and their use for heterologous expression. Nucleic Acids Res. 22, 5767-5768 (1994). 
21. Garí, E., Piedrafita, L., Aldea, M. \& Herrero, E. A set of vectors with a tetracycline-regulatable promoter system for modulated gene expression in Saccharomyces cerevisiae. Yeast Chichester Engl. 13, 837-848 (1997).

22. Nagai, T. et al. A variant of yellow fluorescent protein with fast and efficient maturation for cellbiological applications. Nat. Biotechnol. 20, 87-90 (2002).

23. Balleza, E., Kim, J. M. \& Cluzel, P. Systematic characterization of maturation time of fluorescent proteins in living cells. Nat. Methods 15, 47-51 (2018).

24. Mateus, C. \& Avery, S. V. Destabilized green fluorescent protein for monitoring dynamic changes in yeast gene expression with flow cytometry. Yeast Chichester Engl. 16, 1313-1323 (2000).

25. Mazo-Vargas, A., Park, H., Aydin, M. \& Buchler, N. E. Measuring fast gene dynamics in single cells with time-lapse luminescence microscopy. Mol. Biol. Cell 25, 3699-3708 (2014).

26. Charvin, G., Cross, F. R. \& Siggia, E. D. A Microfluidic Device for Temporally Controlled Gene Expression and Long-Term Fluorescent Imaging in Unperturbed Dividing Yeast Cells. PLOS ONE 3, e1468 (2008).

27. Wu, X.-L. et al. Genome-wide landscape of position effects on heterogeneous gene expression in Saccharomyces cerevisiae. Biotechnol. Biofuels 10, 189 (2017).

28. Bai Flagfeldt, D., Siewers, V., Huang, L. \& Nielsen, J. Characterization of chromosomal integration sites for heterologous gene expression in Saccharomyces cerevisiae. Yeast 26, 545-551 (2009).

29. Crook, N. C., Freeman, E. S. \& Alper, H. S. Re-engineering multicloning sites for function and convenience. Nucleic Acids Res. 39, e92-e92 (2011).

30. Curran, K. A., Karim, A. S., Gupta, A. \& Alper, H. S. Use of expression-enhancing terminators in Saccharomyces cerevisiae to increase mRNA half-life and improve gene expression control for metabolic engineering applications. Metab. Eng. 19, 88-97 (2013).

31. Gordon, A. et al. Single-cell quantification of molecules and rates using open-source microscopebased cytometry. Nat. Methods 4, 175-181 (2007).

32. Lee, M. E., DeLoache, W. C., Cervantes, B. \& Dueber, J. E. A Highly Characterized Yeast Toolkit for Modular, Multipart Assembly. ACS Synth. Biol. 4, 975-986 (2015).

33. Mao, X., Hu, Y., Liang, C. \& Lu, C. MET3 Promoter: A Tightly Regulated Promoter and Its Application in Construction of Conditional Lethal Strain. Curr. Microbiol. 45, 37-40 (2002).

34. Lohr, D., Venkov, P. \& Zlatanova, J. Transcriptional regulation in the yeast GAL gene family: a complex genetic network. FASEB J. 9, 777-787 (1995).

35. Labbé, S. \& Thiele, D. J. [8] Copper ion inducible and repressible promoter systems in yeast. in Methods in Enzymology vol. 306 145-153 (Elsevier, 1999).

36. Svaren, J. Transcription factors vs nucleosomes: regulation of the PH05 promoter in yeast. Trends Biochem. Sci. 22, 93-97 (1997).

37. Becskei, A., Kaufmann, B. B. \& van Oudenaarden, A. Contributions of low molecule number and chromosomal positioning to stochastic gene expression. Nat. Genet. 37, 937-944 (2005).

38. Benzinger, D. \& Khammash, M. Pulsatile inputs achieve tunable attenuation of gene expression variability and graded multi-gene regulation. Nat. Commun. 9, 3521 (2018). 
39. Motta-Mena, L. B. et al. An optogenetic gene expression system with rapid activation and deactivation kinetics. Nat. Chem. Biol. 10, 196-202 (2014).

40. Brophy, J. A. N. \& Voigt, C. A. Principles of genetic circuit design. Nat. Methods 11, 508-520 (2014).

41. Ratna, P. \& Becskei, A. Construction of cis-Regulatory Input Functions of Yeast Promoters. in Yeast Genetic Networks (ed. Becskei, A.) vol. 734 45-61 (Humana Press, 2011).

42. Krebber, A., Burmester, J. \& Plückthun, A. Inclusion of an upstream transcriptional terminator in phage display vectors abolishes background expression of toxic fusions with coat protein g3p. Gene 178, 71-74 (1996).

43. Song, W., Li, J., Liang, Q. \& Marchisio, M. A. Can terminators be used as insulators into yeast synthetic gene circuits? J. Biol. Eng. 10, 19 (2016).

44. Carey, L. B., van Dijk, D., Sloot, P. M. A., Kaandorp, J. A. \& Segal, E. Promoter Sequence Determines the Relationship between Expression Level and Noise. PLoS Biol. 11, e1001528 (2013).

45. Sharon, E. et al. Probing the effect of promoters on noise in gene expression using thousands of designed sequences. Genome Res. 24, 1698-1706 (2014).

46. Bar-Even, A. et al. Noise in protein expression scales with natural protein abundance. Nat. Genet. 38, 636-643 (2006).

47. Gasch, A. P. et al. Genomic Expression Programs in the Response of Yeast Cells to Environmental Changes. Mol. Biol. Cell 11, 4241-4257 (2000).

48. Godard, P. et al. Effect of 21 Different Nitrogen Sources on Global Gene Expression in the Yeast Saccharomyces cerevisiae. Mol. Cell. Biol. 27, 3065-3086 (2007).

49. Crabeel, M. et al. General amino acid control and specific arginine repression in Saccharomyces cerevisiae: physical study of the bifunctional regulatory region of the ARG3 gene. Mol. Cell. Biol. 5, 31393148 (1985).

50. Morgan, D. O. The cell cycle: Principles of control. (Published by New Science Press in association with Oxford University Press ; Distributed inside North America by Sinauer Associates, Publishers, 2007).

51. Barik, D., Ball, D. A., Peccoud, J. \& Tyson, J. J. A Stochastic Model of the Yeast Cell Cycle Reveals Roles for Feedback Regulation in Limiting Cellular Variability. PLOS Comput. Biol. 12, e1005230 (2016).

52. Oikonomou, C. \& Cross, F. R. Rising Cyclin-CDK Levels Order Cell Cycle Events. PLoS ONE 6, e20788 (2011).

53. Wäsch, R. \& Cross, F. R. APC-dependent proteolysis of the mitotic cyclin Clb2 is essential for mitotic exit. Nature 418, 556-562 (2002).

54. Amon, A. Closing the cell cycle circle in yeast: $\mathrm{G} 2$ cyclin proteolysis initiated at mitosis persists until the activation of G1 cyclins in the next cycle. Cell 77, 1037-1050 (1994).

55. Rahi, S. J., Pecani, K., Ondracka, A., Oikonomou, C. \& Cross, F. R. The CDK-APC/C Oscillator Predominantly Entrains Periodic Cell-Cycle Transcription. Cell 165, 475-487 (2016).

56. Skotheim, J. M., Di Talia, S., Siggia, E. D. \& Cross, F. R. Positive feedback of G1 cyclins ensures coherent cell cycle entry. Nature 454, 291-296 (2008).

57. Daniel Gietz, R. \& Woods, R. A. Transformation of yeast by lithium acetate/single-stranded carrier DNA/polyethylene glycol method. in Methods in Enzymology vol. 350 87-96 (Elsevier, 2002). 
58. Silverman, B. W. Density Estimation for Statistics and Data Analysis. (Routledge, 2018). doi:10.1201/9781315140919.

59. Synthetic Complete (SC) Medium. Cold Spring Harb. Protoc. 2016, pdb.rec090589 (2016).

\section{Supplementary information}

Supplementary table 1: Plasmids used in the study

\begin{tabular}{|c|c|c|c|c|c|}
\hline Plasmid & Backbone & Insert & $\begin{array}{l}\text { Restriction } \\
\text { enzymes } \\
\text { used for } \\
\text { the } \\
\text { cloning }\end{array}$ & $\begin{array}{l}\text { Bacterial } \\
\text { selection } \\
\text { marker }\end{array}$ & Source \\
\hline pVG9 & pCL10 & $\begin{array}{c}\text { pC120 - yEVenus - CLN2 } \\
\text { PEST - ADH1t }\end{array}$ & $\begin{array}{c}\text { PacI and } \\
\text { BamHI }\end{array}$ & Ampiciline & $\begin{array}{l}\text { This } \\
\text { study }\end{array}$ \\
\hline pVG10 & pCL10 & $\begin{array}{c}\text { GALLpr - yEVenus - CLN2 } \\
\text { PEST -ADH1t }\end{array}$ & $\begin{array}{c}\text { PacI and } \\
\text { BamHI }\end{array}$ & Ampiciline & $\begin{array}{l}\text { This } \\
\text { study }\end{array}$ \\
\hline pVG11 & pCL10 & $\begin{array}{c}\text { [GAL1pr-pCL120-GAL1pr] } \\
\text { - yEVenus - CLN2 PEST - } \\
\text { ADH1t }\end{array}$ & $\begin{array}{c}\text { PacI and } \\
\text { BamHI }\end{array}$ & Ampiciline & $\begin{array}{l}\text { This } \\
\text { study }\end{array}$ \\
\hline pVG45 & pCL10 & $\begin{array}{c}\text { CUP1pr - yEVenus -CLN2 } \\
\text { PEST-ADH1t }\end{array}$ & $\begin{array}{c}\text { PacI and } \\
\text { BamHI }\end{array}$ & Ampiciline & $\begin{array}{l}\text { This } \\
\text { study }\end{array}$ \\
\hline pVG46 & pCL10 & $\begin{array}{c}\text { PHO5pr - yEVenus - CLN2 } \\
\text { PEST -ADH1t }\end{array}$ & $\begin{array}{l}\text { BsWI and } \\
\text { BamHI }\end{array}$ & Ampiciline & $\begin{array}{l}\text { This } \\
\text { study }\end{array}$ \\
\hline pVG47 & pCL10 & $\begin{array}{c}\text { tetOpr - yEVenus - CLN2 } \\
\text { PEST -ADH1t }\end{array}$ & $\begin{array}{c}\text { PacI and } \\
\text { BamHI }\end{array}$ & Ampiciline & $\begin{array}{l}\text { This } \\
\text { study }\end{array}$ \\
\hline pVG48 & EZL105 & PGK1pr - rtTA - CYCt & $\begin{array}{l}\text { NheI and } \\
\text { XhoI }\end{array}$ & Ampiciline & $\begin{array}{l}\text { This } \\
\text { study }\end{array}$ \\
\hline pVG49 & pCL10 & $\begin{array}{c}\text { GAL1pr - yEVenus - CLN2 } \\
\text { PEST -ADH1t }\end{array}$ & $\begin{array}{c}\text { PacI and } \\
\text { BamHI }\end{array}$ & Ampiciline & $\begin{array}{l}\text { This } \\
\text { study }\end{array}$ \\
\hline pVG50 & pCL10 & $\begin{array}{c}\text { ADH1t - tetOpr - yEVenus } \\
\text { - CLN2 PEST - ADH1t }\end{array}$ & $\begin{array}{c}\text { PacI and } \\
\text { BamHI }\end{array}$ & Ampiciline & $\begin{array}{l}\text { This } \\
\text { study }\end{array}$ \\
\hline pVG88 & pCL10 & $\begin{array}{c}\text { ARG3pr - yEVenus - CLN2 } \\
\text { PEST -ADH1t }\end{array}$ & $\begin{array}{c}\text { PacI and } \\
\text { BamHI }\end{array}$ & Ampiciline & $\begin{array}{l}\text { This } \\
\text { study }\end{array}$ \\
\hline EZL105 & - & PGK1pr - EL222 - CYCt & - & Ampiciline & 4 \\
\hline pVG52 & pVG35 & $\begin{array}{c}\text { pC120 - CLB2kd - yEVenus } \\
\text {-ADH1t }\end{array}$ & $\begin{array}{l}\text { XbaI and } \\
\text { SapI }\end{array}$ & Ampiciline & $\begin{array}{l}\text { This } \\
\text { study }\end{array}$ \\
\hline pCL10 & - & $\begin{array}{c}\text { MET3pr - yEVenus-CLN2 } \\
\text { PEST-ADH1t }\end{array}$ & - & Ampiciline & $L P B S$ \\
\hline
\end{tabular}

Supplementary table 2: Yeast strains used in the study

\begin{tabular}{|c|c|c|}
\hline Strain & $\begin{array}{c}\text { Mating type } \\
\text { (n. d. }=\text { not } \\
\text { determined) }\end{array}$ & Genotype \\
\hline
\end{tabular}




\begin{tabular}{|c|c|c|}
\hline yVG408 (met3.3) & a & ura3:MET3pr - yEVenus - CLN2 PEST - ADH1t:URA3 \\
(single copy)
\end{tabular}

Supplementary note 1: Media composition

\section{Media used for induction experiments}

Standard synthetic complete media without methionine (SC-Met) ${ }^{59}$ was used as the basis for other media, with modifications specific for each promoter detailed below. We used $2 \%$ glucose (D), $3 \%$ raffinose (R), or 3\% glucose $(G)$.

\section{Media for MET induction experiments}

Non-inducing: SCD+10x Met $(1 \mathrm{x}$ Met $=0.02 \mathrm{~g} / \mathrm{mL})$. Non-inducing condition: SCD-Met.

\section{Media for CUP1pr induction experiments}

Non-inducing condition: To make SCD-Met-Cu${ }^{2+}$ we used yeast nitrogen base without copper (Formedium, UK). Inducing condition: SCD-Met-Cu${ }^{2+}$ with $\mathrm{CuSO}_{4}$ added $(0.3 \mathrm{mM})$. 
Non-inducing condition: To make SCD-Met-Pi ( $\mathrm{Pi}$ - inorganic phosphate) we used yeast nitrogen base without phosphates (MP Biomedicals, France). Inducing condition: SCD-Met.

Media for LIP and GLIP induction experiments

Media for both conditions are the same, SCD-Met. Light inducible promoters were induced with the microscope's diascopic LED light.

Media for GAL1pr and GALLpr induction experiments

Non-inducing condition: SCR-Met. Inducing condition: SCRG-Met.

Media for tetOpr and t-tetOpr induction experiments

Non-inducing condition: SCD-Met. Inducing condition: SCD-Met with doxycycline added $(10 \mu \mathrm{M})$.

Media for ARG3pr induction experiments

Non-inducing condition: SDC-Met+10xArg (1x Arg = $0.02 \mathrm{~g} / \mathrm{L}$ of L-arginine monohydrochloride). Inducing condition: SCD-Met-Arg.

Supplementary note 2: DNA sequences of the promoters

Promotors were cloned between BamHI and PacI restriction sites, unless otherwise specified.

pVG9: LIP (5 EL222 binding sites + minimal promoter)

GGATCCTACGTGAGTTCGCCAGCTTCGAGTAGGTAGCCTTTAGTCCATGCGTTATAGGTAGCCTTT AGTCCATGCGTTATAGGTAGCCTTTAGTCCATGCGTTATAGGTAGCCTTTAGTCCATGCGTTATAG GTAGCCTTTAGTCCATGCTTAAGAGACACTAGAGGGTATATAATGGAAGCTCGACTTCCAGCTTG GCAATCCGGTACTGTTGGTAAAGCCACCGCGGCCGCTAAAATCTTAATTAA

pVG10: GALL promoter

GGATCCGGGACAGCCCTCCGAAGGAAGACTCTCCTCCGTGCGTCCTCGTCTTCACCGGTCGCGTTC CTGAAACGCAGATGTGCCTCGCGCCGCACTGCTCCGAACAATAAAGATTCTACAATACTAGCTTT TATGGTTATGAAGAGGAAAAATTGGCAGTAACCTGGCCCCACAAACCTTCAAATGAACGAATCA AATTAACAACCATAGGATGATAATGCGATTAGTTTTTTAGCCTTATTTCTGGGGTAATTAATCAGC GAAGCGATGATTTTTGATCTATTAACGGATATATAAATGCAAAAACTGCATAACCACTTTAACTA ATACTTTCAACATTTTCGGTTTGTATTACTTCTTATTCAAATGTAATAAAAGTATCAACAAAAAAT TGTTAATATACCTCTATACTTTAACGTCAAGGAGAAAAAACCCCGGATTCTATTAATTAA

pVG11: GLIP (5 EL222 binding sites + GAL1 promoter without upstream activation sequence)

GGATCCGGTACCCCCCTCGAGGAATTTTCAAAAATTCTTACTTTTTTTTTGGATGGACGCAAAGAA GTTTAATAATCATATTACATGGCATTACCACCATATACATATCCATATACATATCCATATCTAATC TTACTTATATGTTGTGGAAATGTAAAGAGCCCCATTATCTTAGCCTAAAAAAACCTTCTCTTTGGA ACTTTCAGTAATACGCTTAACTGCTCATTGCTATATTGAAGTGCGGCCGCGGGAGATCTTCGCTAG CCTCGAGTAGGTAGCCTTTAGTCCATGCGTTATAGGTAGCCTTTAGTCCATGCGTTATAGGTAGCC TTTAGTCCATGCGTTATAGGTAGCCTTTAGTCCATGCGTTATAGGTAGCCTTTAGTCCATGAAGCT TAGACACTAGAGGGACTAGACCGTGCGTCCTCGTCTTCACCGGTCGCGTTCCTGAAACGCAGATG TGCCTCGCGCCGCACTGCTCCGAACAATAAAGATTCTACAATACTAGCTTTTATGGTTATGAAGA 
GGAAAAATTGGCAGTAACCTGGCCCCACAAACCTTCAAATGAACGAATCAAATTAACAACCATA GGATGATAATGCGATTAGTTTTTTAGCCTTATTTCTGGGGTAATTAATCAGCGAAGCGATGATTTT TGATCTATTAACAGATATATAAATGCAAAAACTGCATAACCACTTTAACTAATACTTTCAACATTT TCGGTTTGTATTACTTCTTATTCAAATGTAATAAAAGTATCAACAAAAAATTGTTAATATACCTCT ATACTTTAACGTCAAGGAGAAAAAACTATATTAATTAA

pVG45: CUP1 promoter

GGATCCTAAGCCGATCCCATTACCGACATTTGGGCGCTATACGTGCATATGTTCATGTATGTATCT GTATTTAAAACACTTTTGTATTATTTTTCCTCATATATGTGTATAGGTTTATACGGATGATTTAATT ATTACTTCACCACCCTTTATTTCAGGCTGATATCTTAGCCTTGTTACTAGTTAGAAAAAGACATTT TTGCTGTCAGTCACTGTCAAGAGATTCTTTTGCTGGCATTTCTTCTAGAAGCAAAAAGAGCGATGC GTCTTTTCCGCTGAACCGTTCCAGCAAAAAAGACTACCAACGCAATATGGATTGTCAGAATCATA TAAAAGAGAAGCAAATAACTCCTTGTCTTGTATCAATTGCATTATAATATCTTCTTGTTAGTGCAA TATCATATAGAAGTCATCGAAATAGATATTAAGAAAAACAAACTGTACAATCAATCAATCAATCA TCACTTAATTAA

pVG46: PHO5 promoter (cloned with BsWI and PacI restriction enzymes since there is a BamHI cutsite inside the $\mathrm{PHO} 5$ promoter)

CGTACGCAATGTTCCTTGGTTATCCCATCGCCAATAATTTTTATTTTTACCACTGTTGAAGAAGCG AAAGAAAAAAAAAGGGAAAATCAAAACATTCCCTGTGCACTAATAGAAGAAAACAAGAGACTC CGTCCCTCTTTAGTGAGAAAATTGACCAGAGATGGTTTTTGTCCATCTTTTCGCAAAAAATTAGTT CTATTTTTTACACATCGGACTGATAAGTTACTACTGCACATTGGCATTAGCTAGGAGGGCATCCA AGTAATAATTGCGAGAAACGTGACCCAACTTTGTTGTAGGTCCGCTCCTTCTAATAATCGCTTGTA TCTCTACATATGTTCTATTTACTGACCGAAAGTAGCTCGCTACAATAATAATGTTGACCTGATGTC AGTCCCCACGCTAATAGCGGCGTGTCGCACGCTCTCTTTACAGGACGCCGGAGACCGGCATTACA AGGATCCGAAAGTTGTATTCAACAAGAATGCGCAAATATGTCAACGTATTTGGAAGTCATCTTAT GTGCGCTGCTTTAATGTTTTCTCATGTAAGCGGACGTCGTCTATAAACTTCAAACGAAGGTAAAA GGTTCATAGCGCTTTTTCTTTGTCTGCACAAAGAAATATATATTAAATTAGCACGTTTTCGCATAG AACGCAACTGCACAATGCCAAAAAAAGTAAAAGTGATTAAAAGAGTTAATTGAATAGGCAATCT CTAAATGAATCGATACAACCTTGGCACTCACACGTGGGACTAGCACAGACTAAATTTATGATTCT GGTCCCTGTTTTCGAAGAGATCGCACATGCCAAATTATCAAATTGGTCACCTTACTTGGCAAGGC ATATACCCATTTGGGATAAGGGTAAACATCTTTGAATTGTCGAAATGAAACGTATATAAGCGCTG ATGTTTTGCTAAGTCGAGGTTAGTATGGCTTCATCTCTCATGAGAATAAGAACAACAACAAATAG AGCAAGCAAATTCGAGATTACCATTAATTAA

pVG47: tetOpr (based on tet operator sequence)

GGATCCAGATCCGCTAGGGATAACAGGGTAATATAGATCAATTCCTCGATCCCTATCAGTGATAG AGAGTCGACAAAGTCGAGTTTCTCGATCGAGACCACTGCATGCATGTGCTCTGTATGTATATAAA ACTCTTGTTTTCTTCTTTTCTCTAAATATTCTTTCCTTATACATTAGGTCCTTTGTAGCATAAATTAC TATACTTCTATAGACACGCAAACACAAATACACACACTAAATTACCGGATCAATTCGGTTAATTA A

pVG49: GAL1 promoter

GGATCCTTTGGATGGACGCAAAGAAGTTTAATAATCATATTACATGGCATTACCACCATATACAT ATCCATATCTAATCTTACTTATATGTTGTGGAAATGTAAAGAGCCCCATTATCTTAGCCTAAAAAA ACCTTCTCTTTGGAACTTTCAGTAATACGCTTAACTGCTCATTGCTATATTGAAGTACGGATTAGA 
AGCCGCCGAGCGGGCGACAGCCCTCCGACGGAAGACTCTCCTCCGTGCGTCCTCGTCTTCACCGG TCGCGTTCCTGAAACGCAGATGTGCCTCGCGCCGCACTGCTCCGAACAATAAAGATTCTACAATA CTAGCTTTTATGGTTATGAAGAGGAAAAATTGGCAGTAACCTGGCCCCACAAACCTTCAAATTAA CGAATCAAATTAACAACCATAGGATGATAATGCGATTAGTTTTTTAGCCTTATTTCTGGGGTAATT AATCAGCGAAGCGATGATTTTTGATCTATTAACAGATATATAAATGGAAAAGCTGCATAACCACT TTAACTAATACTTTCAACATTTTCAGTTTGTATTACTTCTTATTCAAATGTCATAAAAGTATCAAC AAAAAATTGTTAATATACCTCTATACTTTAACGTCAAGGAGTTAATTAA

pCL10: MET3 promoter

GGATCCTTTAGTACTAACAGAGACTTTTGTCACAACTACATATAAGTGTACAAATATAGTACAGA TATGACACACTTGTAGCGCCAACGCGCATCCTACGGATTGCTGACAGAAAAAAAGGTCACGTGAC CAGAAAAGTCACGTGTAATTTTGTAACTCACCGCATTCTAGCGGTCCCTGTCGTGCACACTGCACT CAACACCATAAACCTTAGCAACCTCCAAAGGAAATCACCGTATAACAAAGCCACAGTTTTACAAC TTAGTCTCTTATGAAGTTACTTACCAATGAGAAATAGAGGCTCTTTCTCGAGAAATATGAATATG GATATATATATATATATATATATATATATATATATATATGTAAACTTGGTTCTTTTTTAGCTTGTG ATCTCTAGCTTGGGTCTCTCTCTGTCGTAACAGTTGTGATATCGTTTCTTAACAATTGAAAAGGAA CTAAGAAAGTATAATAATAACAAGAATAAAGTATAATTAACATTAATTAA

pVG88: ARG3 promoter

GGATCCTCTTCTAAGAAAAAATATTTAGATCATATTATTTTAGATAACCGAGACATCGTTAGCAA CCATGACTCCAGTAAACAAAAATTCAAGATCCAGAATATTTTGAACTCGACCTTCTAACATTACG CTCCTTCGTATTACTCATTCAGCTCTTCCTCTGATAGCAGTGAATTTTCGAGGGTCACGTCGTGAC TCATATGCTTTCTTGTTCCGTTTCGTTTCGAGATGACAAAAAACTGGTCATTTTTTCCGTTAAGTGC AACTCACAGCAGTATCGGCCGCTGAGAAATGCCCGGACAAATTTTTTTGAGCCGGATTGGTCACC GTTTCTTTCTTCGGCGCGGCTTCCCATTCCCGTCCATCCAAAAAAATCTACCTATATAAATCGACT TTTCACCTCTAAAGGCAGTTTATTCCTTGTATGTCCTTTAAGTACAGTTAATAACGAGCAATTTTTT TTTTTTTTTTTAGCCATCTACCCATCAACTTGTACACTCGTTACCTTAATTAA

Supplementary note 3: Single-copy integration search procedure

To verify that cells have only one copy of the promoter-FP construct, we devised a PCR-based procedure that allowed us to distinguish between single and multiple copy insertions. For this, we designed two pairs of primers $\mathrm{p}$ fwd /p rev ( $\mathrm{p}=$ plasmid) and $\mathrm{g}$ fwd /g rev ( $\mathrm{g}=$ genome). Both pairs of primers amplify the region containing the URA3 gene with the difference that $\mathrm{p}$ primers anneal to the plasmid backbone only while g primers anneal to the yeast genome only. Since the plasmids were cut inside the plasmid's URA3 gene prior to insertion, in case of single copy integrations, the p pair of primers should not give a PCR amplicon (Supplementary figure 1). On the other hand, if the plasmid is integrated in the genome in multiple copies, the p pair of primers will produce an amplicon. With this test, we screened for colonies that showed no PCR product with the p primer pair. To be certain that the lack of amplification was not due to low DNA quality or potential oversight in the PCR reaction, we also performed PCR using $\mathrm{g}$ fwd/p rev and $\mathrm{p} \mathrm{fwd/g} \mathrm{rev} \mathrm{pair} \mathrm{of} \mathrm{primers,} \mathrm{which} \mathrm{should} \mathrm{show} \mathrm{amplification} \mathrm{of} \mathrm{the} \mathrm{DNA} \mathrm{regardless} \mathrm{of} \mathrm{the} \mathrm{copy}$ numbers of the construct. We then only used the strains that showed amplification with $\mathrm{g}$ fwd/p rev and $\mathrm{p}$ fwd/g rev and no amplification with $\mathrm{p}$ fwd/p rev pair of primers. This also confirmed that the construct is integrated in the URA3 locus. We repeated this analysis at least twice with independent PCR reactions performed on independent genomic DNA extractions. 
Plasmid with URA3 marker

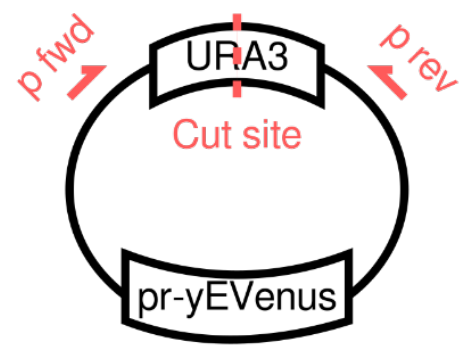

Genomic URA3 locus

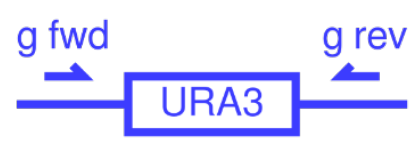

Single-copy integration

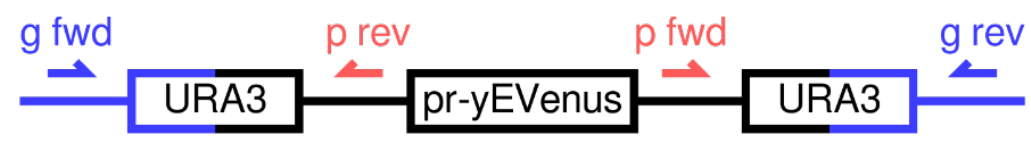

Multiple-copy integration

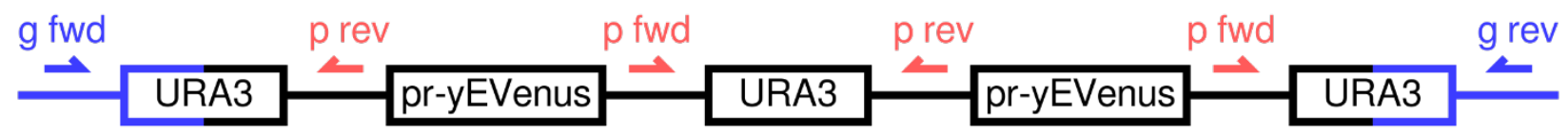

Supplementary figure 1 . Single and multiple-copy integrations can be distinguished by a PCR-based strategy. 